\title{
Perspective of Elderly on Age-Friendliness of Services Provided in Primary Healthcare Centers of Dubai Health Authority: Focus Groups Discussions
}

\author{
Tamer Mohamed Farid ${ }^{*}$, Amal Mohamad Saleh Abdulrahim Al Jaziri' ${ }^{1}$, Soha Abdelziz Abdelal1, \\ Anood Jamal Alshaali' ${ }^{1}$, Manal Mohammad Omran Taryam ${ }^{2}$, Nahed Abdul Khaleq Monsef ${ }^{3}$, \\ Afaf Abdullatif Al Hashemi ${ }^{4}$, Hamidah Saleh Al Shaibany ${ }^{4}$
}

${ }^{1}$ Elderly Care Unit, Health Affairs Department, Primary Health Care Services Sector, Dubai Health Authority, Dubai, UAE

${ }^{2}$ Primary Health Care Services Sector, Dubai Health Authority, Dubai, UAE

${ }^{3}$ Health Affairs Department, Primary Health Care Services Sector, Dubai Health Authority, Dubai, UAE

${ }^{4}$ Health Centres Department, Primary Health Care Services Sector, Dubai Health Authority, Dubai, UAE

Email: ^Tamer.M.Farid@gmail.com

How to cite this paper: Farid, T.M., Al Jaziri, A.M.S.A., Abdelal, S.A., Alshaali, A.J., Taryam, M.M.O., Monsef, N.A.K., Al Hashemi, A.A. and Al Shaibany, H.S. (2018) Perspective of Elderly on Age-Friendliness of Services Provided in Primary Healthcare Centers of Dubai Health Authority: Focus Groups Discussions. Advances in Aging Research, 7, 132-144.

https://doi.org/10.4236/aar.2018.76011

Received: September 25, 2018 Accepted: November 17, 2018 Published: November 20, 2018

Copyright $\odot 2018$ by authors and Scientific Research Publishing Inc. This work is licensed under the Creative Commons Attribution International License (CC BY 4.0).

http://creativecommons.org/licenses/by/4.0/

\begin{abstract}
Primary Health Care Services Sector (PHCSS) of Dubai Health Authority (DHA) provides many services dedicated to seniors. However, there have been no studies to date that consulted the seniors themselves regarding these services. Thus, this study was conducted to investigate the age-friendliness of outpatient clinic's services provided in PHCSS from perspective of elderly service recipients. Methods: Three focus groups were designed. The focus groups included receivers of care (senior patients and caregivers for senior patients) and healthcare providers (physicians, nurses, administrators and medical record officers). The discussed topics in the focus groups were based on WHO's recommended three domains of improvement for an age-friendly Primary Health Care (PHC): Information, education and training, community-based health care management systems, and the physical environment.
\end{abstract}

Keywords

Focus Group, Dubai, Elderly, Primary Healthcare, Age-Friendly, WHO

\section{Introduction}

In 2002, the UN released the Madrid plan of action on aging [1]. In order to 
achieve one of its three main priorities, namely Advancing health and well-being into old age, it set objectives to develop and strengthen primary health-care services to meet the needs of older persons, and promote their inclusion in the process. Concomitantly, the WHO organized focus groups in five countries between June and September 2002, involving 300 elder participants in 36 groups, and 50 healthcare providers in 8 groups to get the views of care receivers and care providers, in order to develop principles that render community health centres more age-friendly. Information gained from the focus groups led to the development of a set of age-friendly principles that are designed to serve as a guide for community-based PHC centres to modify management and clinical services, staff training and environments to better fit the needs of their older patients. The Age-friendly Principles address three major areas [2]:

1) Information, Education, Communication and Training;

2) Health Care Management Systems;

3) The Physical Environment.

These three major areas of development were used in some researches to as domains of assessment of extent of adaptation of healthcare services to needs of elderly in PHC [3] [4] [5] and even hospitals [6].

The PHC health centers (HCs) of DHA are both JCI [7] and ISO [8] accredited, and the administration is continuously surveying the patients in clinics for opinions in services provided. However, to date there has been no comprehensive and structured discussion with elderly in the clinics on their perspective of services provided.

The emirate of Dubai is a member of the federation of seven emirates of United Arab Emirates. It's inhabited by 2,446,675 permanent residents, of whom, 59,977 [9] aged 60 years or more (2.45\%). Majority of population is concentrated in the city of Dubai.

\section{Patient's Journey Inside Primary Health Care Centers}

Governmental health services provided in the Emirate of Dubai are provided through the Ministry Of Health (the federal health authority) and the Dubai Health Authority DHA (the local health authority) DHA is the prime provider within Dubai. The PHC Service Sector (PHCSS) the division of DHA that provides the PHC in 12 Health Centers (HCs) across the Emirate of Dubai, each serving a geographical catchment area. All HCs has Family Physician (FP) clinics, a laboratory, a pharmacy and basic radiology unit. Some HCs can have an additional number of other medical specialty clinics. Patients within the geographical catchment area of the HC get the services through the FP clinic, who can refer these patients to other specialties' clinics inside the same HC, in other HCs or to DHA hospitals. Before any outpatient visit, a nurse performs assessment for the patient and guide the patient to the required steps. Referral to other specialty or services outside the HC required additional arrangements by the medical record officer, to communicate with the destination service, and the patients receives a text message by phone. On the other hand, ordering medica- 
tions, and tracking visits history are managed online directly by the treating physician through the DHA intra-net. Visit notes were still recorded in patients' paper file until end of 2017, when electronic medical record gradually replaced the paper file.

In the geriatric medicine clinic or any other specialty service, the patient is usually scheduled for follow up visit after 3 months, which is primarily used for refill of prescription for chronic medications, beside evaluation of health condition.

Geriatric medicine services in DHA: Elderly receive care from geriatric medicine physicians through;

1) Elderly care unit provides a team of specialists that covers outpatient specialized geriatric clinic (clinics for geriatric medicine, memory, osteoporosis, and falls and rehabilitation), conducts home visits and formulates guidelines for clinical care relevant to health of elderly.

2) Long Term Care and rehabilitation at seniors' happiness center.

3) A hospital-based team for consultation and treatment of admitted elderly patients in Rashid hospital.

All healthcare services for citizens of UAE are covered by government insurance, and elderly receive them for free.

\section{Subjects and Methods}

Two convenience samples of seniors attending the HCs and caregivers for home-bound elderly were selected. A third purposive sample of health care staff working in HCs was selected. Each of these three groups participated in one of three focus groups; one for male senior patients (and their caregivers), one for female senior patients (and their caregivers), and the last group for health care professionals. Caregivers were invited into the focus groups to express the experience of home bound and demented older patients.

\subsection{Inclusion Criteria for Participant in Patients and Caregivers Focus Groups}

Seniors had to be 60 years of age or more, mentally competent, attended their respective health center's clinic from 1 August 2015 to 1 August 2016, had at least 3 years of follow up in HCs (to accumulate enough experience with the services provided), and were willing to participate in focus group.

Caregivers had to be the principal caregivers of dependent seniors, and were personally in contact with health care system.

Participants had to be Arabic-speaking, as it is the communication language in the focus groups, and they are vast majority of seniors in PHC.

\subsection{Inclusion Criteria for Participant Healthcare Staff Focus Groups}

Participants had to be Arabic-speaking, as it is the communication language in the focus group. 


\subsection{Conducting Focus Groups}

Three focus groups (males, females and healthcare staff) were carried out on three successive days, $(25,26$ and 27 October 2016) during working hours in a health centers; patient focus groups were held in Nadd Al-Hamar HC, while healthcare staff were interviewed in Al-Mizhar HC.

All meetings were moderated by director Elderly Care Unit, in PHCSS of DHA. During the meetings, the participants' experiences with services in HCs were discussed. The discussion content was based on the three major areas of action for an Age-friendly PHC, as suggested by the WHO's guide [10]: 1) information, education and training, 2) community-based health care management systems, 3) the physical environment. Discussion of each of these three topics was covered in a 45 minutes session. The moderator in each session raised a question about issues relevant to the domain, and encouraged the participants to elaborate their opinions and experiences. Sessions were video-recorded and responses were transcribed to maintain descriptive validity of results. To ensure interpretative validity, themes were supported by participants' own words. Transcripts were reviewed and analyzed by two members of the research team. Recurring themes within and among groups were identified.

\subsection{Limitations}

1) Restriction of participation to Arabic-speaking patients.

2) Non-probability sample.

\section{Ethical Approval}

Study was approved by the Health Affairs Department of the PHCSS.

\section{Results}

Convenient samples of 10 participants; 3 male seniors (aged 62, 66, and 82), one care giver (daughter) for a male senior (aged 82). Two had a high school education and the other two finished college. The participants in female group were 4 female seniors (aged 63, 65, 71, and 76), and two caregivers (daughters) for female seniors (aged 76 and 80). One of them was illiterate, two had a high school education and two had finished college

The focus group of health care professionals was formed of 10 members; two HC administrators, one medical records officer, two geriatricians, four family physicians, and an outpatient clinic nurse.

Participants comments were covered under the following themes:

1) Inadequate clinic interview

2) Communication barriers

3) Clinical geriatric skills

4) Waiting time and priority for seniors

5) Referral procedure

6) Physical environment of health center (HC) 
7) Social services

8) Escort in clinic

9) Signage; value and readability

\subsection{Inadequate Clinic Interview}

Seniors were frustrated over the frequent missing of their paper files, and the lack of review of their past medical history from the file. Some of them had to repeat the medical history in many visits.

Seniors and administrative officers reported that only geriatricians comprehensively review the medical condition and medication interactions.

"No body directs us to solve our problems except the geriatricians, they were explaining to us and directed us to the available resources". (both patients groups)

Treatment plan was preserved across different clinics, although the paper file was sometimes missing (especially if attending at $\mathrm{HC}$ other than their home $\mathrm{HC})$.

Pharmacists are kind, but list of medications for geriatricians and their duration are restricted by system regulations, which burdens home-bound seniors as they need to referrals to multiple specialties to get their regular medicines. (an elder lady)

Seniors receive health education in specialty clinics rather than FP clinics. The given duration for interviewing any patient in the family medicine clinic was set at 12 minutes for any patient regardless of age. FPs found it too restricted for the multiple comorbidities of elderly, beside tracking the usual online and paper forms. Seniors and physicians understood that there was a lack of proper health education to the patients due to limited time slot,

"We explain the therapeutic options to them, but not what is happening (disease details)". (a family physician)

Staff members suggested that a dedicated health educator for seniors should carry this mission.

\subsection{Communication Barriers}

Patients-particularly females-expressed that many of the medical staff in the HCs-particularly nurses-are non-Arabic speaking, while the majority of seniors are Arabic-speaking. Language barrier was most evident with nurses while handling clinical history and complaint.

We understand what the non-Arabic nurses say, because they know basic words. (all patients)

But language barrier was more a problem for other Arabic-speaking health center members when they dealt with non-Arabic speaking seniors.

"We frequently request interpreters (for non-Arabic speaking patients) from the working staff in $\mathrm{HC}$ "

(a family physician).

Actually, overcoming the language barrier was one the main reasons that se- 
niors were accompanied by an escort in their visits to the HC.

"I need an interpreter, and I call my daughter, who escorts me." (an elder lady).

Orientation was needed on culturally sensitive issues and cognitive and psychological changes with aging, and ageism; one family physicians stated that some staff members still stereotype seniors as cognitively impaired and discuss medical condition with family or escort despite their mental competency.

"It's not common for physicians or nurses to introduce themselves to seniors." (an elder lady)

"Doctors don't identify themselves, but I get their names from the reception". (one male patient)

Caregivers complained that phones of reception desks at some centers have long queues, and patients or their family members failed to inquire about appointments.

Seniors don't know many of the services they can use, aside from the ones they are used to, though they know that they are printed, partially because many of them can't read. In addition, for the same reason, they didn't know many of the operational information of the HCs such as presence of 24 hour services or available specialty clinics in the HCs. The receptionists thought that they were at the best position to orient seniors on their rights and available services, but they didn't have a guide for all services provided in each specialty clinic. Seniors had never been consulted regarding the design of services relevant to them, and they appreciated so much the chance given in the focus group.

\subsection{Clinical Geriatric Skills}

Both seniors and HC professional (including non-medical members) reported the need for further training on clinical geriatric skills, and that only geriatricians consolidated the clinical picture, and assessed seniors' condition comprehensively, while other discipline tended to be restricted within their scope.

"Geriatricians are the ones that consolidate the medical condition and the treatment plan for the elderly, other disciplines only offer treatment in a limited scope". (a medical records officer)

The multiple comorbidities and atypical presentations of disease in the elderly were the major challenges that faced family physicians.

"Some physicians expect elderly not to understand treatment instructions, while they offer all instructions to younger patients". (a family physician)

Therefore, orientation of FPs was needed on clinical topics such as geriatric syndromes and theoretical topics such as ageism and age-related changes in body systems.

Despite believing that family physicians needed further training on clinical geriatric skills, seniors trust the DHA physicians' care more than private physicians' care, as the patients noticed that the management of government targeted their health improvement not a financial gain. In addition, patients viewed the professional competency of both government and private physicians as equally high. 


\subsection{Waiting Time and Priority for Seniors}

Seniors confirmed that the administrative staff showed respect to them but complained that they didn't give them their due priority in queues.

"The healthcare team (especially the medical records officers) don't give me my due priority over others". (an elder lady)

"We didn't know that there is a mechanism to advance the ticket for elderly on top of queue for clinic appointment booking" (an elder lady).

All seniors complained of the waiting time inside the HCs; it was too long, and it was partially due to incoordination of file handling. There was a wide variations in estimations for the cause of long waiting time that covered the whole patient journey inside the outpatient clinic of health center. The paper file was missing in many occasions, with the need to issue a new one with no previous notes.

Seniors considered priority as an integral part of respecting them, and it's part of the tradition.

"Respecting priority for elderly is a local tradition that the local citizens respect, but not most of the expats." (one male patient)

\subsection{Referral Procedures}

Seniors find the procedures of referral too challenging, because they were variable depending on the intended service, and they were not clear.

"Many online referral requests are not executed, and the most frequent cause is errors during filling online forms by referring physician". A medical records officer

"Sometimes I am not clearly told the reason for referral or the expected outcome of the interview in the destination clinic". (an elder lady)

Seniors find internal referrals to hospitals' clinics from within the hospital easier than referral from HCs, as all it needs is referral slip, without complex communications and lengthy steps.

\subsection{Physical Environment of Health Center (HC)}

The route to HC is unobstructed and easy for repeated visitors, but unknown and difficult to first time visitors due to lack of guide signs. This is encountered when they are referred to another HC for the first time. Seniors in general don't use public transportation because they have their private cars. But those come by bus suffer the long distance between HC and nearest bus stops.

In general, the inside physical environment of most HCs is well accepted by seniors. They are clean, well-ventilated, well-lit and designed to serve most of seniors' needs. Nevertheless, waiting area is the most annoying to seniors; some HCs has metal chairs that are too rigid for those suffering back problems and too cold. Also, waiting area become full sometimes and seniors may need to sit somewhere else until a seat becomes available. Waiting areas are not well designed to accommodate wheel chairs, which leaves seniors on wheel chairs 
parked outside them, or in the corridors. Safety and fall prevention is taken in consideration and pieces of furniture are numbered, inspected and organized daily.

\subsection{Social Services}

"Thokhr" cards entitles seniors for priorities and discounts in some governmental offices. It's not useful in HC.

"I knew about available social services only when the social worker phoned me". (an elder lady)

Seniors find physicians in HCs cost-sensitive when it comes to prescribing therapies that are not covered by insurance, and always explain about domiciliary devices, but nobody lists social and home care available for seniors.

\subsection{Escort in Clinic}

Many seniors rely on their escort inside HC to plays many important roles, starting from transportation, assistance with mobility, translation, and organizing all subsequent steps of the clinical visit inside the HC and referral to other OPCs. All focus groups-especially ladies-supported implementation of a "concierge" service inside HCs.

\subsection{Signage: Value, Readability}

Staff identification; it's not common for physicians to introduce themselves to seniors, and there are no name boards for the physicians. The seniors had to ask the receptionist or the registration officers about the name of the physician!

Seniors disregard the signs in the HC, and ask the attending staff for direction and procedures because it's easier for them, and some of them cannot read.

"When I travel to Germany I follow the signs (in the Health Centers). The sings in the local HCs are not inferior, but asking staff is easier". (one male patient).

However, the signs were important to the non-Arabic speaking visitors to HC.

\section{Discussion}

The participation rate was relatively lower than expected, this may be explained-even partially-by the participants. Note that it is was the first time they were invited for discussion of services provided in HC. As it is a new experience, the invited elderly had no background about the benefits, the plan, the proceedings, the privacy and other issues that may represent burden to them.

During the focus groups sessions, there were differences in the relative priorities of discussion among different groups. Healthcare professionals concentrated more on the need for training on geriatric medicine skills, while patients focused on waiting area in the health center, under estimation of their social status as senior members of the society, and the value of their escorts to their tour in the health center. Ladies valued the discussion of their medical condition more than 
men did. One issue was universally present across all groups, and this was the language and communication barrier.

There was an anonymous agreement between the three separate groups on

1) Inadequacies in clinic interview due to administrative load.

2) Ambiguity of referral procedures.

3) Need for training on management of chronic comorbidities in seniors

So, many issues were not as relevant to elderly as had originally been expected; including the signage design, accessibility of health center to public transportation, language barrier,

\subsection{Inadequacies in Clinic Interview}

The three separate focus groups agreed on the impact of procedural process in experience of elderly attending the health centers, both during the clinical visit and in the referral after the visit.

This seems to be due to the structure of the system rather than training of physicians or limited facilities in the health centers, since seniors expressed that they trust the professional level of physicians in health centers, and viewed them on a par to their counterparts in private clinics, and the need for training on geriatric medicine skills was actually expressed more among healthcare team rather than patients themselves.

The DHA has shifted towards a fully electronic medical file management system, which terminated the use of paper file. This was completed in the fourth quarter of 2017. Starting from January 2018, no paper files are need to be transported to the clinic at which the patient is attending to review notes from previous visits, as the latter are included in the electronic medical record. This is expected to improve the efficiency in clinical interview, by decreasing time consumed for documentation, among other benefits as well.

In a survey of perceived barriers to care for elderly on Medicare in US [11], the most reported barrier (by 32.9\% of respondents) was lack of response by their doctor. Lack of satisfaction with care has also been found to be associated with more symptoms and lower medication compliance in patients [12] whereas greater satisfaction has been associated with better outcomes [13].

\subsection{Communication Barriers}

Arabic-speaking healthcare worker was found to be one of top three factors encouraging patients to utilize PHC in Riyadh, Saudi Arabia [14], a culturally and economically comparable city to Dubai. Despite the fact that nurses in DHA are able to communicate basic Arabic language, this might have not been sufficient to handle the complex needs of the multiple chronic comorbidity of elderly diseases. This high proportion of non-Arabic speaking nurse in Arab gulf countries is common; in a study at Eastern Provenience of Saudi Arabia, 61.5\% of nurses in PHC were non-Arabic [15], and patient participants in the study expressed insufficient language competency and cultural knowledge as well. 
Language barrier is essentially present in healthcare system in any multi-ethnicity society as USA [16]. Informal (Ad hoc) interpreters, such as family members or untrained members of the support staff, are commonly called in to help communicating with the patient. The DHA has implemented a system for solving language barrier that relies on medical staff members that are familiar with medical terms. This is called the "privilege list". The health center enrolled its staff members that are native speakers of the commonly spoken languages in Dubai on the list, to act as a interpreters when needed. Nevertheless, elderly resorted to their escort, who is usually bilingual. These escorts are commonly a family member, a professional caregiver or a private nurse.

The DHA has been moving towards intelligent software solutions [17], that include dynamic webpage, health status monitoring applications, electronic health reminder. All these customizable solutions can help relieving phone queues and language barriers. Similar smart phone applications and online services are being used on daily basis in all Dubai government transactions such as managing bills, issuing licenses, booking appointment. The newly introduced electronic medical record management system SALAMA [18] (from EIPIC), allowed secure patient access to his/her data-including appointments-without the need for contacting the receptionist in HCs.

\subsection{Waiting Time and Priority for Seniors}

Seniors viewed their seniority as an integral part of Emirati culture and values, and this seniority entails giving them priority. Thus, elders expected their requests to receive priority over other age groups, and this entails immediate attention and should result in minimal delay till service is delivered.

Part of feeling disrespected was due the perception of loss of the seniority rights because the priority booking for elderly was done automatically, and it was not visible to them, except that their fast track numbering was different from the serials of the normal track. Therefore, it may be satisfying to them to expose the steps of advancing their requests at the beginning of the queue to affirm their priority.

\subsection{Clinical Geriatric Skills}

The reports of need of training of family physicians on geriatric medicine skills met a report of family physicians in an another study on the three major domains of difficulty that they encounter when managing elderly in the outpatient: 1) medical complexity and chronicity, 2) personal and interpersonal challenges, and 3) administrative burden [19].

Causes for deficient geriatric clinical skills among non-geriatricians,

Despite the rapid expansion of percentage of elderly in population worldwide, this is not properly reflected in the exposure in undergraduate medical training or the number of post-graduate programs in geriatrics. Lack of national curricula and objectives for geriatric training. In the TeGeMe-II Study of WHO for un- 
dergraduate exposure to geriatric education, about half of all countries assessed (53\%) have national regulations for medical school curricula, and only $41 \%$ of the curricula mentioned geriatrics in some way [20]. This is one reason for shortage of geriatricians world wide; for example, even in USA, while there were estimated 20,053 geriatricians needed in 2016 [21] only 6874 (34.28\%) geriatricians were licensed [22] excluding osteopathic geriatricians [23], with a shortage of 13,176 geriatrician $(65.71 \%)$.

\subsection{Referral Procedures}

The referral process in the old "SAM" electronic system was challenging to elderly; due the diversity of services that they were referred to, and the variations of some steps depending on the service. With the introduction of the new "SALAMA electronic system, the referral process became unified, streamlined and paperless.

\subsection{Physical Environment of Health Center (HC)}

Despite the fact that the design of the HC was age-friendly [5], the elderly could not tolerate waiting due to several reasons; discomfort because of there skeletal problems, their need to visit the rest room more often, intolerance to cold weather inside the $\mathrm{HC}$, and their expectation for a faster service as a part of the cultural respect they enjoy. This long waiting time was the main reason for them to seek private clinics or drop the follow up visits. In a study for satisfaction of elderly attendants of HC clinics in Asir [24], Saudi Arabia, despite they were generally satisfied with the services, the second most frequent cause for their dissatisfaction was long waiting in HC (46.4\% of elderly surveyed).

\subsection{Escort in Clinic}

The escort to the elderly in HC played a pivotal role. This escort could be a family member, a professional caregiver or the driver. The escort could handle almost all HC visit activities on behalf of the patient, besides transferring wheelchair-bound patients. In such sense, the escort played a positive role as the deputy of the patient, and had a positive impact on patient's healthcare. However, the escort may have some drawbacks; as the patients relied on him/her, they disregard the sings and educational materials in the center, because they trusted the escort for solving the problems. Also, in many occasions, the escort is registered his/her phone to receive reminder SMS(s) for the patient appointment, and disregard the reminder SMS later on it since it was not pertinent for him/her. Thus, the elderly patients may eventually became shielded from outreach of the HC that had been designed to orient them to the services available in the $\mathrm{HC}$, health promotion programs, their rights, or the use of the fast track system in booking for elderly. This fueled their frustration due to the belief that they were not given the due respect and attention. The escort may also disregarded preventive services as he/she focused more on active problems. 


\section{Acknowledgements}

Special thanks for the advice, assistance and review in the preparation of this manuscript to:

Dr. Moulham Saleh Ashtar, Head of clinical effectiveness office, Health Affairs Department, Primary Health Care Services Sector, Dubai Health Authority, Dubai, United Arab Emirates.

Dr. Salah Ahmed Mohamed Elbadawi, Consultant Public Health, PHC Chief Executive Officer Office, Primary Health Care Services Sector, Dubai Health Authority, Dubai, United Arab Emirate.

\section{Conflicts of Interest}

The authors declare no conflicts of interest regarding the publication of this paper.

\section{References}

[1] United Nations (2002) Political Declaration and Madrid International Plan of Action on Ageing. New York, 39-40.

[2] World Health Organization (2012) Towards Age-Friendly Primary Health Care. World Health Organization, France, 8.

[3] Woo, J., Mak, B. and Yeug, F. (2013) Age-Friendly Primary Health Care: An Assessment of Current Service Provision for Older Adults in Hong Kong. Health Services Insights, 6, 69-77. https://doi.org/10.4137/HSI.S12434

[4] Alhamdan, A., Alsshammari, S., Al-Amoud, M., Hameed, T., Al-Muammar, M., Bindawas, S., Al-Orf, S., Mohamed, A., Al-Ghamdi, E. and Calder, P. (2015) Evaluation of Health Care Services Provided for Older Adults in Primary Health Care Centers and Its Internal Environment. Saudi Medical Journal, 36, 1091-1096. https://doi.org/10.15537/smj.2015.9.11789

[5] Farid, T., AlJaziri, A., Taryam, M., Monsef, N., Buharoun, A., Elbadawi, S. and Ashtar, M. (2017) Dubai Primary Health Care Centers Conformation to WHO Age-Friendly Primary Healthcare Recommendations. Advances in Aging Research, 6, 83-92. https://doi.org/10.4236/aar.2017.66009

[6] Ahmadi, A., Seyedin, H. and Fadaye-Vatan, R. (2015) Towards Age-Friendly Hospitals in Developing Countries: A Case Study in Iran. Health Promotion Perspectives, 5, 42-51. https://doi.org/10.15171/hpp.2015.006

[7] Joint Commission International (2017) JCI-Accredited Organizations. United Arab Emirates-Primary Health Care.

http://www.jointcommissioninternational.org/about-jci/jci-accredited-organization s/?c=United\%20Arab\%20Emirates\&a=Primary\%20Care\%20Program

[8] Dubai Health Authority (2016) Eleven Primary Health-Care Centers Run by DHA Receive Six ISO Certification.

https://www.dha.gov.ae/en/DHANews/Pages/DHANews953853650-09-10-2016.aspx

[9] Dubai Statistics Center (2015) Statistical Year Book, Chapter 1: Population and Vital Statistics, Table (01-04).

https://www.dsc.gov.ae/Publication/\%D8\%A7\%D9\%84\%D8\%A8\%D8\%A7\%D8\%A 8\%20\%D8\%A7\%D9\%84\%D8\%A3\%D9\%88\%D9\%84-\%20\%D8\%A7\%D9\%84\%D8\% B3\%D9\%83\%D8\%A7\%D9\%86.pdf 
[10] Kalache, A., Hoskins, I. and Mende, S. (2004) Towards Age-Friendly Primary Health Care. World Health Organization, Geneva.

[11] Fitzpatrick, A., Powe, N., Cooper, N., Ives, D. and Robbins, J. (2004) Barriers to Health Care Access among the Elderly and Who Perceives Them. American Journal of Public Health, 94, 1788-1794. https://doi.org/10.2105/AJPH.94.10.1788

[12] Harris, L., Luft, F., Rudy, D. and Tierney, W. (1995) Correlates of Health Care Satisfaction in Inner-City Patients with Hypertension and Chronic Renal Insufficiency. Social Science \& Medicine, 41, 1639-1645. https://doi.org/10.1016/0277-9536(95)00073-G

[13] Alazri, M. and Neal, R. (2003) The Association between Satisfaction with Services Provided in Primary Care and Outcomes in Type 2 Diabetes Mellitus. Diabetic Medicine, 20, 486-490. https://doi.org/10.1046/j.1464-5491.2003.00957.x

[14] Saeed, A. and Mohamed, B. (2002) Patients' Perspective on Factors Affecting Utilization of Primary Health Care Centers in Riyadh, Saudi Arabia. Saudi Medical Journal, 23, 1237-1242.

[15] Aldossary, A., Barriball, L. and While, A. (2013) The Perceived Health Promotion Practice of Nurses in Saudi Arabia. Health Promotion International, 28, 431-441. https://doi.org/10.1093/heapro/das027

[16] Flores, G. (2006) Language Barriers to Health Care in the United States. The New England Journal of Medicine, 355, 229-231. https://doi.org/10.1056/NEJMp058316

[17] Dubai Health Authority (2017) The Dubai Health Authority Exhibited Its Latest Innovative Medical Services, Initiatives and Smart Apps at the Dubai International Government Achievements Exhibition 2017. https://www.dha.gov.ae/en/DHANews/Pages/DHANews8141948-05-04-2017.aspx

[18] Dubai Health Authority (2017) DHA Launches the Final Phase of the Salama Electronic Medical Record System.

https://www.dha.gov.ae/en/DHANews/Pages/DHANews885910175-19-11-2017.asp x

[19] Adams, W., McIlvain, H., Lacy, N., Magsi, H., Crabtree, B., Yenny, S. and Sitorius, M. (2002) Primary Care for Elderly People: Why Do Doctors Find It So Hard? The Gerontologist, 42, 835-838. https://doi.org/10.1093/geront/42.6.835

[20] Keller, I., Makipaa, A., Kalenscher, T. and Kalache, A. (2002) Global Survey on Geriatrics in the Medical Curriculum. World Health Organization, Geneva.

[21] Fried, L. and Hall, W. (2008) Leading on Behalf of an Aging Society. The Journal of the American Geriatrics Society, 6, 1791-1795. https://doi.org/10.1111/j.1532-5415.2008.01939.x

[22] American Board of Medical Specialties (ABMS) (2016) 2015-2016 ABMS Board Certification Report. Geographic Distribution of ABMS Member Board Diplomates by Subspecialty Certificate. http://www.abms.org/media/131568/2015-16-abmscertreport.pdf

[23] Scheinthal, S., Kramer, J. and Morales-Egizi, L. (2016) Appendix 2: American Osteopathic Association Specialty Board Certification. The Journal of the American Osteopathic Association, 116, 263-266. https://doi.org/10.7556/jaoa.2016.051

[24] Mahfouz, A.A., Al-Sharif, A.I., El-Gamal, M.N. and Kisha, A.H. (2004) Primary Health Care Services Utilization and Satisfaction among the Elderly in Asir Region, Saudi Arabia. Eastern Mediterranean Health Journal, 10, 365-371. 